\section{Getting a Turnout: The Plight of the Organizer}

\author{
Experiences in \\ promoting a \\ computer conference ${ }^{1}$
}

by Chuck Humphrey

The biggest problem seems to be just building up the momentum to get people started. I started the CIPS conference about 6 months ago, signing up about 20 people. Now there are more like 30 signed up but only about 10 who use it and less than that who contribute much. However, that seems to be a fact of computer conference in general. ${ }^{2}$

This quotation expresses some of the frustration that computer conference organizers face as they attempt to initiate and nurture a conference. The existence of a computer conference hinges upon the level of involvement of its members; and yet, achieving an active membership appears

\footnotetext{
'Supported by a SSHRC Grant No.421-830013 ${ }^{2}$ A personal electronic message received from Jon H.Nightingale, December 14, 1983.
}

to be one of the most difficult tasks confronting the conference organizer.

Jon Nightingale's experience in beginning a national computer conference for the executive of the Canadian Information Processing Society (CIPS) is similar to my experience in organizing DataLink, a national computer conference for Canadian data libraries and archives. Out of a group of about thirty members, roughly only ten participants can be described as being active. Some of the factors which affect small group participation, in general, will undoubtedly apply to computer conferences of this size. However, unique barriers to participation do exist for computer conferences and the discussion below addresses some of these.

Types of computer conferences

The level of activity among computer conferences seems to vary according to the task of the conference and its membership restrictions. Using the dimensions of task orientation and scope of membership, a computer conference can be characterized as being one of four types. Examples of these are shown in Figure 1. (Ed. nole: fiqures and tables are gathered logether at end of article)

Having participated in conferences fitting each of these classifications, I have generally found the General-Task/Open-Membership conferences to be the most active, while the Specific-Task/Closed-Membership groups have tended to have the most sporadic participation. ${ }^{3}$ The following discussion describes

${ }^{3}$ The "level of participation" or "activity" of a conference can be defined in a number of ways. Below, "activity" is measured in terms of connect time. Yet another way to described the level of participation in a conference is to identify active and passive participants. This too is mentioned below. 
a case study of this latter type.

\section{DataLink: a case study}

During the 1983 Learned Societies in Vancouver, a one-day workshop was sponsored jointly by the Federal Department of Communications and the Machine Readable Division of the Public Archives. ${ }^{4}$ This meeting brought together a number of the principals involved in the funding, generation, analysis and preservation of machine readable data files (MRDF). Among the participants were spokesmen for granting agencies, data producers. researchers, and data depositories.

Out of this meeting two issues seemed to converge. First, the need for a set of guidelines which advise in the preparation and deposit of MRDF was expressed by all participants. Secondly, a call was made to form an ongoing computer conference to encourage discussion among the staff of Canadian data libraries and archives. $^{5}$ At that time, no Canadian forum existed for professionals in this field.

Encouraged by a supportive response in Vancouver, a proposal was submitted to the SSHRCC requesting support for a computer conference which would exist primarily to draft a set of MRDF standards. ${ }^{6}$ Therefore, the

\footnotetext{
${ }^{4} \mathrm{~A}$ summary of the session, "Towards a Canadian Electronic Heritage: The Vancouver 6 June Consultation," is available from Harold Naugler, Director, Machine Readable Archives, Public Árchives Canada.

${ }^{5}$ L. Ruus, W. Watkins and C. Humphrey, "A Proposal for a Computer Conference for Managers of Canadian Data Libraries and Archives," (Vancouver, mimeo), 1983. ${ }^{6} \mathrm{C}$. Humphrey and W. Watkins, "A Funding Request to Implement the 'Proposal for a Computer Conference for Managers of Canadian Data Libraries and Archives'," August 1983.
}

orientation of the conference was clearly defined from the beginning as being task specific.

Membership was restricted to practitioners who we knew to work with MRDF on a regular basis. In April 1984, twenty-seven invitations were mailed; twenty replied indicating a willingness to participate. Eleven additional invitations were sent in June of which seven of these agreed to participate.? Membership by nature was closed. Thus, DataLink clearly fits the task-specific/closed-membership conference type in Figure 1.

Special problems: group maintenance

One concept used to analyze group behavior is the task-maintenance dimension. ${ }^{8}$ Task processes deal with the group accomplishing its organizational purpose, while maintenance processes function to hold the group together and to secure the members' commitment to the group's task.

Organizing a computer conference where most of the members do not know each other and where the members are spread across a continent poses special maintenance problems. How will the level of participation in a computer conference be affected by the low level of interpersonal contact among the members? How does an organizer conduct team building exercises under these conditions?

\footnotetext{
${ }^{7}$ One more person joined in late August bringing the total membership to 29 including the organizer.

${ }^{8}$ The task-maintenance dimension is one aspect of L. Richard Hoffman's hierarchical model of group behavior. See L. Richard Hoffman, Improving the Problem-Solving Process in Managerial Groups," Improving Group Decision Making in Organizations (Academic Press, 1982). p. $95-125$.
} 
Membership maintenance: an ad hoc experiment

The question about how the level of participation in the computer conference would be affected by members not seeing each other first-hand was examined in an ad hoc experiment. The proposed date for starting Datalink was chosen to coincide with the 1984 annual meeting of the International Association for Social Sciences Information Service and Technology (IASSIST), which serves as an international forum for producers, users and archivists of machine readable data. Since the IASSIST conference was held in Ottawa in May, it was seen as an ideal oppotunity to gather the members of DataLink in an in-person workshop. Thus, participants could attend a major conferences in their professional field and also meet to initiate DataLink.

While not all of the original twenty members of DataLink were also able to attend the IASSIST workshop, eleven members did meet. Since two groups resulted from the division between those who attended and those who did not attend, a quasi-experimental design presented itself for group comparisons (see Table 1,). Thus the in-person workshop at the IASSIST conference functioned as an experimental condition, while the nine members not attending the workshop formed a control group. Those who were invited to join DataLink after the meeting in Ottawa formed yet a second control group.

The obvious weakness in this ad hoc design is the non-random assignment of paricipants to the experimental and control groups. Any differences in activity level between these groups could be attributed to uncontrolled factors separating these members who attended the workshop from those who did not attend. One major uncontrolled factor was the amount of a priori commitment by members to Datalink. In fach an argument can be made that the cost of time and travel to Ottawa alone indicate a relatively strong commitment by the experimental group.

The dependent variable measuring the activity level of participants is their average monthly connect time with DataLink. Since one group of participants was not invited until June, average monthly connect time in minutes was used rather than total minutes. These calculations were based through December 1984. As mentioned in an earlier footnote, this is only one measure of participation. Using total connect time combines both between the two control groups, although these figures still remain about half the number of average monthly minutes spent by members of the experimental group.

The distribution of connect time for each group is skewed as shown in Figure 2. The experimental group has the widest midspread and the only outlier of the three distributions. By transforming the dependent variable to the natural $\log$ scale, the distributions become more symmetric (see Figure 3). A prominent feature of Figure 3 is the wide degree of variability in the experimental group. While this group has the highest median, it also has the widest spread of connect times. The first control group however has the narrowest midspread, although it is skewed toward the smaller connect times.

Standard non-parametric tests of the medians and ranks however reveal no statistically significant differences among the three groups (see Table 3). The size of each group is small and two groups have wide midspreads; therefore these test results are not particularly surprising. Nevertheless, a trend is evident in the summary figures of Table 3 for the counts of those above, below and tied with the overall media. Two-thirds of the experimental group are above the overall median, while only 30 and 33 per cent of the first and second control groups, respecuively, are above this figure. 
The results are inconclusive whether attending the in-person meeting actually increased the level of participation by these members. While a larger proportion of the experimental group had connect times greater than the overall median, the variability of connect times was also the greatest in this group. There were some members who attended the in-person meeting who subseqently participated minimally in DataLink. In addition, the non-random assignment of members to the three groups seriously prevents attributing an effect to the in-person meeting. A priori commitment may be a more important explanatory variable.

\section{Other barriers to participation}

While other obstacles to participation were not examined like the above question about the importance of personal contact among the members, several barriers to participation have been identified. The discussion below describes some of these obstacles and offers suggestions for minimizing their impact.

\section{Start-up barriers}

One of the most difficult tasks confronting an organizer is getting each member to use the conferencing system for the first time. Short of sitting down with each member and insisting that they $\log$ on the system in your presence, that is, coercion, the incentives for motivating people to begin using the conference are difficult to define. With DataLink, the invitation letter clearly expressed the commitment that was expected of them:

"What will be your commitment":
First, you will be asked to join the computer conference as an active participant. This means that you will be expected to check the conferencc activities on at least a weekly basis and to contribute to the discussions within the conference.

Secondly, you are encouraged to attend the 1984 IASSIST conference to be held in Ottawa from 15 - 17 May. During these meetings a special gathering will be held to initiate the computer conference and to train participants in the use of the conferencing software."

Nevertheless, people are volunteering their time and simply making expectations clear does not necessarily close the gap between intention and action.

Thercfore, one of the primary concerns for the organizer is to increase the likelihood that once they do $\log$ on the system, their first conferencing encounter will be a positive experience. It is difficult enough getting a novice even to try a conferencing system, but all the more difficult to keep that person on the system if their first conferencing experience is bad.

There are a number of precautions that can be taken to minimize bad first attempts. Probably the most preventive measure that can be taken is for the organizer to be present when members are first joining the conference, although this is far too often impractical. With Datalink, we were able to get most of those who attended the workshop in Ottawa to join the conference with a consultant present.

Another precautionary step is to register members in the conferencing system before hand, including directory files for electronic mail systems. Since conference registration occurs only the first time the system is used, pre-registering participants removes a non-recurring feature. Therefore, expectations 
about how the system behaves upon entering the program arc not different from the first time to the next. Also, registration can requirc a fair amount of explanation which can be avoided entirely.

Forum, the hosi system under which DataLink exists, places the user in a general conference mode rather than in a specific conference. However, it is much better to place users into a specific conference that to leave them in general mode. The user simply faces less ambiguity as to where they are located during their conferencing session. The members of DataLink all had a file initialized prior to their first conferencing session which placed them automatically into DataLink.

Documentation: more than just a cliche

While emphasizing the importance of documentation has become a computing industry cliche, printed documentation is essential for computer conference members who are at distances greater than a local phone call to a consultant. The documentation however must be useful. Most computer systems are not short on documentation, rather they are short on useful documentation. Poorly written documentation unfortunately can be as big an obstacle to the user as too little documentation.

Two types of documents were used with DataLink: a tutorial guide and a reference manual. The latter document already cxisted; however, the tutorial guide had to be written. ${ }^{10}$

\footnotetext{
${ }^{9}$ Alan Ballard and Jon Nightingale, "A Computer Conferencing Program," (University of British Columbia, mimeo), April 1984. ${ }^{10}$ Charles Humphrey, "DataLink Forum: An Introduction to the DataLink Computer Conference" (University of Alberta, mimeo), May 1984.
}

The style which was followed applies some of the idcas from script theory in social psychology. ${ }^{11}$ In its simplest form, this style consists of providing a script that details how to act. Documentation written in this manner requires less cognitive attention by the user than concept-oriented documentation, that is, there is a smaller processing load on the reader. Manipulating and understanding concepts become secondary to getting the user simply to match their interaction with the system to a script.

Script-like documentation should also include many examples about how a conferencing session should appear. Again, the focus is toward getting the user to match their expcrience to the script. I have found many users of statistical packages to employ this type of heuristic when doing data analysis. They will locate an example which is close to their problem, replicate the setup of the example and then fine-tune the example to their problem. Applying this style to the DataLink tutorial seems to have been effective since several of the participants have made favorable comments about the document.

Surviving the low points: summer's heat can cool the conference

The months of May and June are not particularly wise choices for beginning a computer conference. If you manage to achieve any momentum during these two months, you are guaranteed to lose this pace in July or August. Figure 5 shows the cumulative number

\footnotetext{
${ }^{11} \mathrm{~A}$ concise summary of script theory is found in Robert Abelson, "Script Processing in Attitude Formation and Decision Making" in J.S. Carroll and J.W. Payne, eds., Cognition and Social Behavior (New York: John Wiley), 1976.
} 
of lines entered in discussions and responses from May to December. A summer plateau reveals that few lines of text were entered during that period. Figure 6 , which displays the total number of discussions and responses for each month, shows a similar relationship. During the month of August, not one entry was made.

In an effort to restart activity in September, all previous conference entries were listed and formatted with a text-processor to produce a newsletter, The DataLink Report. The participants were each mailed a copy of this newsletter along with a cover letter encouraging them to participate in DataLink. Several members commented that they liked receiving the newsletter and some said that they preferred reading the items in print rather than on-line. Consequently, two newsletters were mailed in October and one each in November and Dcccmber. Only the most recent contributions were reported in each issue of the newsletter.

One further item mailed with each newsletter was a listing of all participants and the date of their last conferencing session. Serving as a crude report card, it was intended to apply a bit of social pressure to check in with DataLink on a more frequent basis. A couple of the members mentioned some slight embarrassment by their placement on the listing, although its total impact seemed negligible. Guilt does not always motivate.

Reviewing conventional beliefs

In the original proposal for DataLink, a number of reasons were given which postulated the uscfulness of a computer conference in bringing together the staff of data libraries and archives. ${ }^{12}$ Most of these arguments were based upon beliefs about how computer conferences should work. In light of the experiences with DataLink over the past eight months, some of these propositions need updating.

The original arguments about the medium fit into three categories: gained efficiencies through the medium, democratic aspects about the medium, and the technological compatibility of the medium with this group. The propositions within each of these categories are discussed below.

The cost advantage mentioned in Figure 7 of getting together a group of people from across the continent are clearly held by this medium. An economy, return airfare from Montreal to Edmonton obtained through a seat sale costs around $\$ 350.00$. Compare this cost to the conferencing expenses incurred by one of our more active participants from Montreal. In November, his conference usage cost $\$ 11.74$, which covered one hour and 43 minutes of connect time during which he entered 173 lines of text and read approximately 500 lines of text. The ratio of this specific monthly usage charge to the cost of the special airfare would pay for 51 hours and 11 minutes of computer conferencing or approximately 30 months of usage similar to his November level of activity. ${ }^{13}$

The second point in Figure 7 argues that the asynchronous nature allows more individual

\footnotetext{
${ }^{12} \mathrm{~L}$. Ruus, W. Watkins and C. Humphrey, "A Proposal for a Computer Conference for Managers of Canadian Data Libraries and Archives," (Vancouver, mimeo), 1983, pp. 3-4. ${ }^{13}$ Of course this assumes that computing charges will remain constant over time. This in fact has not been the case. More recently, time-sharing costs have been dropping while

telecommunication charges have been slight rising. However, new competition among networks is appearing on the horizon and then telecommunication charges may even begin to drop.
} 
freedom for choosing when to participate in group's activity. Unfortunately, the asynchronous aspect of computer conferencing can lead to non-synchronous behavior! Rather than using the flexibility of the medium to schedule a convenient time for conferencing, the person simply may not participate at any time. While in-person meeting imposes a time and place to meet, a computer conference relies on each member to establish for themselves a time to participate. Such self-discipline has been shown by only a few participants in DataLink. Table 4 shows that over half of the members do not $\log$ on the system each month, while only around 25 per cent seem to participate on a regular basis.

The fourth and fifth points in Figure 7 deal with the computer conference maintaining a permanent record of its transactions and the slowness of getting out traditional newsletters, respectively. It was noted above that copies of the discussions in DataLink were printed as newsletters. In fact, several of the members indicated that they wanted to receive the printed version of the conference. It may be that rather than replacing the traditional newsletter, the computer conference will function as a means of producing newsletters slightly faster.

The openness created by a computer conference, as summarized in the list of democratic aspects of the medium in Figure 8 , does not however necessarily lead to a wider discussion of issues. A couple of the members have mentioned that they have felt insufficiently knowledgeable to comment on the material in DataLink. Consegently, they have not entered into discussions; but a worse consequence has been that they do not introduce any new topics which they have the confidence to discuss. Thus the scope of the discussions within the conference is narrowed by this implicit norm to say nothing.

Furthermore, providing the opportunity to comment does not necessarily lead to meaningful contributions. The norm of keeping silent for fear of saying something wrong has an opposing counterpart: the habitual commentator. They indiscriminately enter a comment on any and all discussions. This has not been a problem in DataLink. However in some conferences where this has happened, organizers have been asked to warn the habitual commentator to refrain from chattering on-line or to face possible expulsion. from the conference.

The technological compatibility arguments in Figure 9 unfortunately fail to acknowledge the complexity introduced by the maze of computing equipment that is brought to bear with a networked conference and the accessibility of these machines to conference members. Some of the participants have reported that terminals are not conveniently available to them at work. They complain about having to leave their desk and immediate work environment to use a terminal in a public setting. Members who are in this situation are infrequent participants. Others have reported problems with communication software on their personal computers. On separate occasions, two have been stymied by full-duplex settings which have prevented them from seeing what they were typing.

Others have noted that they would rather prepare their entries on their own system and then transfer the text to the conference. This is easily possible for some where host to host connections exist. For example, file transfer is possible between the University of British Columbia's central system and the University of Alberta's mainframe, where DataLink resides. A couple of members prepare their entries on IBM $\mathrm{PCs}$ and then use microcomputer communication software to upload files and to capture and download text in return. However, the majority of the members do not have this flexibility and are required for the most part to communicate at the level of an ordinary teletype. 
Three other complaints have arisen about computing facilities. First, the system at the University of Alberta is not available to users from 2:00 to 8:00 am mst. This prevents participants in the Eastern Time zone from connecting with the conference until 10:00 am at the earliest thcir time (although they may use the system until 4:00 am their time). The second complaint is also related to convenient connect times. Datapac, the network connection to the University of Alberta's system, has been intolerably slow during peak work hours. Some users report that the best response time occurs after 4:00 pm The third complaint has occursed about the response time on the University of Alberta's mainframe. This normally has not been a problem; but there were about three weeks during the end of the 1984 Fall Semester when the system's response time was diminished during work hours. Peak loads at that time would have been near 600 simultaneous terminal users on the system. All three factors tend to narrow the choice of times when participants can connect with DataLink. Thus the asynchronous aspect of the medium becomes secondarily controlled by the time constraints placed by the network and host system.

Closing comment: the load on the organizer

The potpourri of experiences and suggestions discussed above point toward the need for an active conference organizer with a task-specific/closed-membership conference. Not only does the organizer of this type of conference have to care for the content of the discussions within the conference, ${ }^{14}$ but a

\footnotetext{
${ }^{14}$ Caring for the text-base of the computer conference generally consists of editing lines that were mangled when entered, converting entries which are all in upper case to mixed case, correcting typographic errors, and maintaining the keyword or index system for the entries.
}

number of maintenance tasks must be done to keep the group functioning.

The trials and tribulations of being a conference organizer can be depressing at times. Where does an organizer go for counselling and encouragement? To a computer conference for organizers, of course! Two conferences along this line have been particularly helpful:

CNFR:ORGANIZER and CRLT:ORGANIZER. ${ }^{15}$ The membership of these two conferences consists of a number of experienced conference organizers who have been very supportive of other organizers in their struggles to keep conferences functioning."

\footnotetext{
${ }^{15}$ The host conferencing system for both is Confer Il. The former is located on Wayne State University's MTS system, while the latter is on the central University of Michigan system.
} 
Getting a Turnout.....figures

Figure 1

Examples of Computer Conferences by Task Orientation and Membership Recruitment

\begin{tabular}{c|c|c}
\hline \multirow{2}{*}{$\begin{array}{c}\text { TASK } \\
\text { ORIENTATION }\end{array}$} & MEMBERSH P RECRUI TMENT \\
\cline { 2 - 3 } Specific & Task Force or Commission & $\begin{array}{c}\text { Working Committee } \\
(\text { e.g., DataLink }\end{array}$ \\
\hline \multirow{2}{*}{ General } & $\begin{array}{l}\text { Expanded Bulletin Board } \\
\text { (e.g., CRLT:MICROSu) }\end{array}$ & $\begin{array}{l}\text { Planning Group } \\
\text { (e.g., MTS:FORUM }\end{array}$ \\
\hline
\end{tabular}

- Datalink is a conference within the Forum system at the University of Alberta.

a CRLT:MICROS and MTS:FORUM are conferences using CONFER II, a product of Advertel Communication Systems, at the University of Michigan.

\section{Figure 2}

Multiple Sample Comparisons of the Average Monthly Minutes Using Datalink

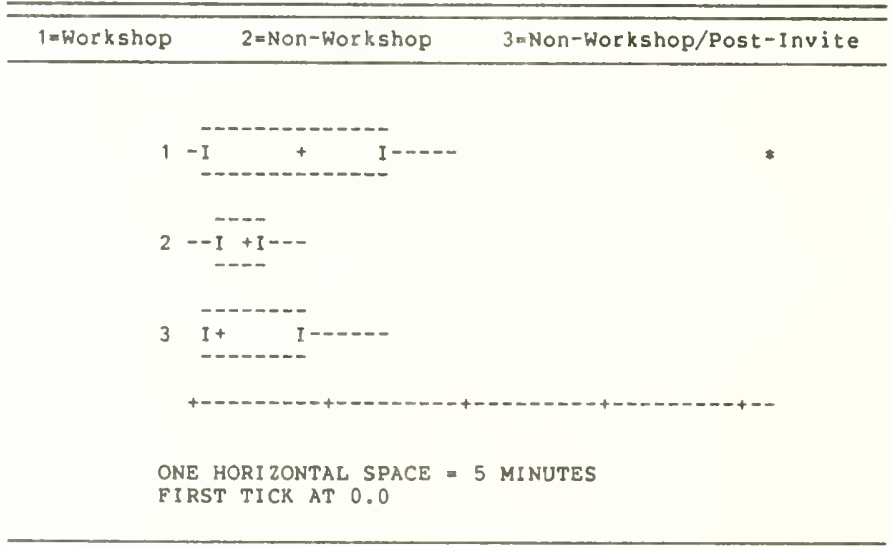




\section{Figure 3}

Multiple Sample Comparisons of the Average Monthly Minutes (LOG) Using Datalink

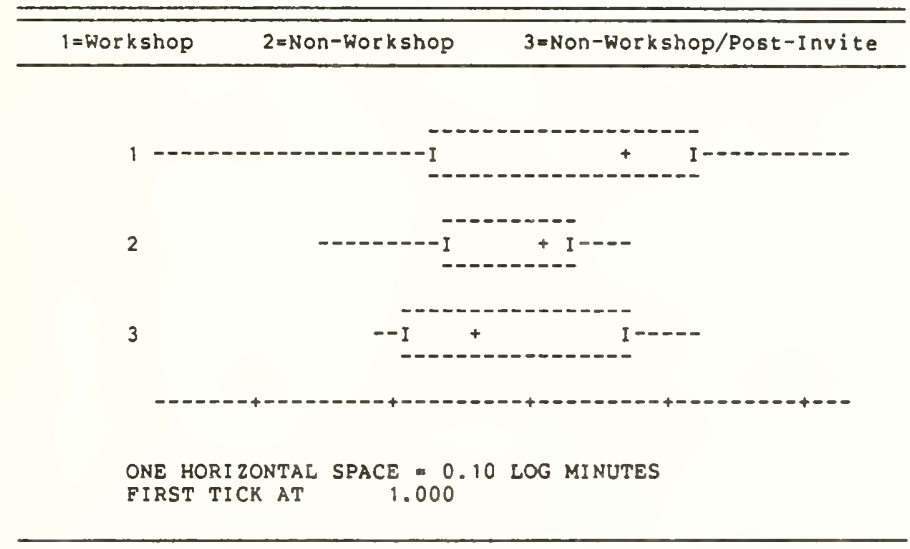

Figure 4

\section{Some DO's and DON'Ts in the Start-Up Stage}

- Do Help the New User Log On the First Time If Possible.

a Do Register Participants Ahead of Time.

D Don't Require the New User to Learn a Feature that They'll Only Use Once, Such as Registration Steps.

- Do Present the System in the Way that It' 11 Respond Time After Time, i.e., Maintain Consistency.

$\square$ Don't Leave Users Guessing where They Are in the Conferencing system.

- Do Provide Script-Level Tutorial Documentation. 


\section{Figure 5}

The Cumulative Number of Lines Entered in

Discussions and Responses in DataLink,

May - December, 1984

No. Lines

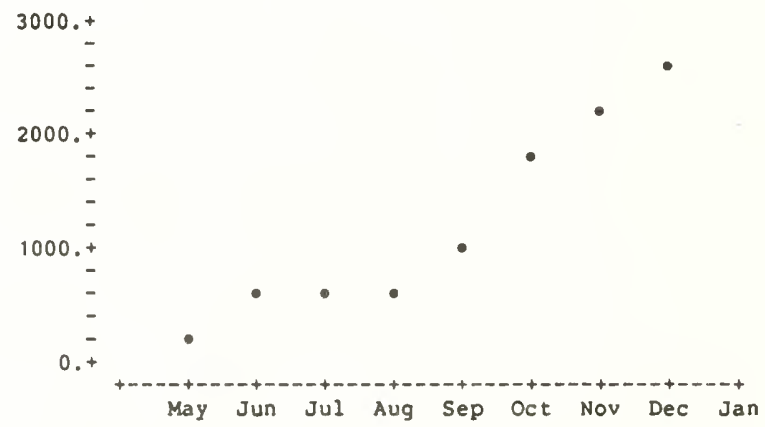

Figure 6

The Monthly Number of Discussions and Responses

Entered In Datalink, May - December, 1984

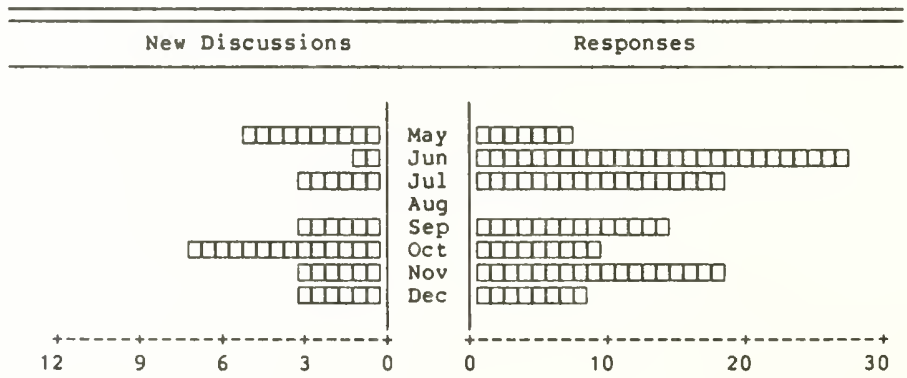


Figure 7

Gained Efficiencies

- [A computer conference] is an inexpensive means of providing ... a common forum or vehicle for discussion. To achieve the same goal through an annual conference, or even a newsletter, would far exceed the costs of supporting a computer conference.

- One of the most attractive features of computer conferencing is the asynchronous nature of the medium. There is no need to schedule a common time for all members to meet, rather, each member participates at his own convenience. This promotes wider participation and completely eliminates the need to arrive at a common timetable to conduct business.

- Comments can be made with more preparation than in a face-to-face meeting, in which it is often impossible to research a topic thoroughly before making comment on unscheduled items.

- Computer conferences maintain a permanent written record of all transactions, eliminating the need for traditional record keeping practices.

- [N]ewsletters are a very slow vehicle for communication.

Figure 8

Democratic Aspects

- [N]o one is excluded from a computer conference for want of travel funds ...

- Newsletters ... seldom reflect the opinions of more than a few people on any specific topic.

- In a computer conference, all participants have an equal opportunity to 'voice' their opinions and to read immediately the replies of others.

- Rarely, in face-to-face meetings, do all present have an opportunity to voice their opinions, for whatever reasons.... In a computer conference no one is excluded from comment. Those who wish to 'say' something have an equal chance to comment. 


\section{Figure 9}

\begin{tabular}{l}
\hline Compatible Technology \\
\hline - Data library staff are aiready 'computer literate'. The \\
computer terminal is a commonplace tool in any data \\
library. Thus, little training or persuasion would be \\
required to introduce participants to the mechanics of \\
conducting the conference. \\
- Each member can participant from wherever a tele- \\
communications connection can be made, whether it \\
be from an office desk or a kitchen table.
\end{tabular}

\section{Table 1}

Quasi-Experimental Design: Non-Random Assignment, Posttest-Only Control Groups

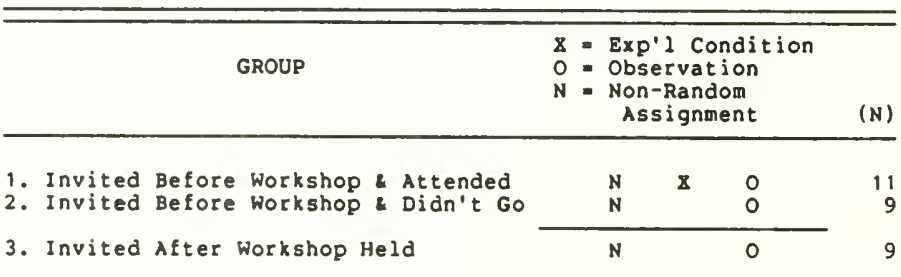

Table 2

Summary Figure Comparisons of the Average Monthly Minutes Using DataLink

\begin{tabular}{lccr}
\hline & \multicolumn{3}{c}{ Group } \\
\cline { 2 - 4 } Summary Statistics & Workshop & Non-Workshop & $\begin{array}{r}\text { Non-Workshop } \\
\text { Post-Invite }\end{array}$ \\
\hline Avg. Monthly Minutes & & & \\
Median & 42.5 & 24.8 & 16.1 \\
Lower Hinge & 11.0 & 12.8 & 8.7 \\
LH-UH Midpoint & 42.2 & 21.1 & 26.4 \\
Upper Hinge & 73.4 & 29.4 & 44.2 \\
Midspread & 62.4 & 16.6 & 35.5 \\
N & 9 & 8 & 6 \\
\hline Avg. Monthly Min. (LOG) & & & 2.71 \\
Median & 3.75 & 3.21 & 2.16 \\
Lower Hinge & 2.40 & 2.49 & 2.98 \\
LH-UH Midpoint & 3.35 & 2.94 & 3.79 \\
Upper Hinge & 4.30 & 3.38 & 1.62 \\
Midspread & 1.90 & 0.89 & 6 \\
N & 9 & 8 & \\
\hline
\end{tabular}


Table 3

Multiple Sample Comparisons of the Average Monthly Minutes Using DataLink

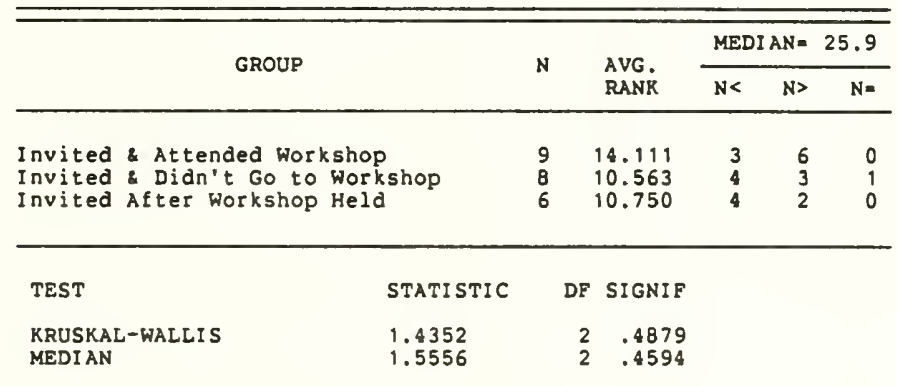

Table 4

The Distribution of Datalink Members by the Number of Monthly Conference Sessions, June - December, $1984^{\circ}$

\begin{tabular}{|c|c|c|c|c|c|c|c|c|c|}
\hline \multirow[b]{2}{*}{ Month } & \multicolumn{8}{|c|}{ Number of Times on the System } & \multirow{2}{*}{$\begin{array}{l}\text { No. of } \\
\text { Memberat }\end{array}$} \\
\hline & None & 1 & -2 & $3-$ & -4 & 5 & -6 & $\begin{array}{l}7 \text { or } \\
\text { More }\end{array}$ & \\
\hline $\begin{array}{l}\text { June } \\
\text { July } \\
\text { August } \\
\text { September } \\
\text { October } \\
\text { November } \\
\text { December }\end{array}$ & $\begin{array}{l}12 \\
13 \\
17 \\
18 \\
16 \\
15 \\
15\end{array}$ & & $\begin{array}{l}5 \\
9 \\
6 \\
1 \\
4 \\
5 \\
4\end{array}$ & $\begin{array}{l}1 \\
0 \\
1 \\
5 \\
2 \\
1 \\
2\end{array}$ & $\begin{array}{l}1 \\
0 \\
1 \\
5 \\
2 \\
1 \\
2\end{array}$ & & $\begin{array}{l}0 \\
2 \\
2 \\
0 \\
0 \\
1 \\
2\end{array}$ & $\begin{array}{l}7 \\
3 \\
2 \\
4 \\
6 \\
6 \\
4\end{array}$ & $\begin{array}{l}25 \\
27 \\
28 \\
28 \\
28 \\
28 \\
27\end{array}$ \\
\hline
\end{tabular}

- The figures for May 1984 were not available.

a Membership changed slightly over the months. There were 20 members at the end of May. One person withdrew and six new members joined in June. Two were added in July and one more started in August. In December, one formally withdrew. 